\title{
A PERCEPÇÃo dO USUÁRIO DA CASA INTELIGENTE: UMA REVISÃO SISTEMÁTICA DA LITERATURA
}

Débora Rosa Nascimento (debora.nascimento@ifmg.edu.br) - PPGEP, Universidade Federal de Santa Catarina (UFSC).

Diego de Castro Fettermann (dcfettermann@gmail.com) - PPGEP, Universidade Federal de Santa Catarina (UFSC).

\section{RESUMO}

O avanço das tecnologias para automação residencial possibilitou o surgimento das casas inteligentes. Cada vez mais, os sistemas de casas inteligente tem sido discutido nas áreas de tecnologia, política e sustentabilidade. Porém, o sucesso de implementação da casa inteligente depende de diversos fatores de aceitação dessa tecnologia por parte dos usuários. Assim, o objetivo deste trabalho é identificar na literatura os fatores que possam ser determinantes no processo de adoção da casa inteligente. Foi utilizada a abordagem de revisão sistemática da literatura. Foram identificados quatro grupos de benefícios promovidos pela casa inteligente: saúde, ambiental, financeiro e psicológico, além de três grandes grupos de barreiras que podem afetar a adoção da casa inteligente: tecnológicas; financeira, ética e legal; e falta de conhecimento e resistência psicológica. Os resultados possibilitam definições de melhores estratégias para a implementação e aceitação desta tecnologia por parte dos usuários.

Palavras chave: Casa inteligente; usuário; revisão sistemática; benefícios, barreiras 


\section{INTRODUÇÃO}

A transformação de produtos e serviços tradicionais em inteligentes desencadeou a utilização de tecnologias que permitem a comunicação entre os dispositivos e outros sistemas (GRAMHANSSEN; DARBY, 2018). Essas tecnologias possibilitaram o surgimento das casas inteligentes, que se tornaram temas centrais nas recentes discussões sobre tecnologia e política e inovação ( DEL RIO et al., 2020). O sistema de casa inteligente vem alcançando novos mercados nos últimos tempos, seja pelo aumento da disponibilidade de recursos digitais (HANNOVER; OSTWESTFALEN-LIPPE; HANNOVER, 2020), seja pela melhoria do conforto e da qualidade de vida dos residentes (LIU, 2021).

O sucesso da implementação de uma casa inteligente e sua plena utilização tende a apresentar uma alta complexidade (DEL RIO et al., 2020). A literatura menciona a presença de diversos fatores para a aceitação da tecnologia de casa inteligente por parte do usuário (e.g., HANNOVER; OSTWESTFALEN-LIPPE; HANNOVER, 2020; MARIKYAN; PAPAGIANNIDIS; ALAMANOS, 2019; DONG et al., 2017). Para que o mercado de casas inteligentes se desenvolva plenamente, é relevante compreender as preferências e necessidades dos clientes (WAN et al., 2017). A partir disso, este artigo busca responder a seguinte questão: quais são os fatores que interferem na aceitação da tecnologia da casa inteligente por parte dos usuários?

A partir desta questão, este artigo tem por objetivo identificar os benefícios promovidos pela casa inteligente e as barreiras do processo de adoção da casa inteligente.

\section{REVISÃO SISTEMÁTICA DA LITERATURA - MÉTODO DE PESQUISA}

A revisão sistemática tem sido usada para identificar estudos que abordam a adoção de casa inteligente (CHAN et al., 2009; MARIKYAN; PAPAGIANNIDIS; ALAMANOS, 2019; SEPASGOZAR et al., 2020). A presente revisão sistemática da literatura utilizou o procedimento proposto por Kitchenham (2004) para o mapeamento do estado da arte sobre a aceitação de casas inteligente por parte dos clientes. O procedimento proposto por Kitchenham (2004) foi inicialmente desenvolvido na área de Engenharia de Sistemas, mas apresenta aplicação disseminada em outras áreas da engenharia, tais como gestão de projetos (MUSAWIR; ABD-KARIM; MOHD-DANURI, 2020), engenharia industrial (KANG; CATAL; TEKINERDOGAN, 2020; SILVA; ANDRADE; FERREIRA, 2020) e inteligência artificial (SPOLAÔR et al., 2020). O processo de revisão sistemática proposto por 
Kitchenham (2004) recomenda três principais fases: planejamento da revisão, condução da revisão e relato da revisão.

$\mathrm{Na}$ fase de planejamento da revisão é desenvolvido o protocolo de revisão. Neste trabalho, para identificar os estudos primários sobre o tema, adoção de casas inteligente, foi determinada como estratégia a definição e validação de palavras-chave, e a definição das bases de dados para realização das buscas. Para definição das palavras-chave foram identificados dois eixos temáticos: "casa inteligente" e "usuário". Para cada um desses eixos, foram definidas as seguintes palavras-chaves: (1) casa inteligente - Smart home; Smart house; Intelligent home; Intelligent house; (2) usuário - adopt*; accept*. As bases de dados utilizadas para pesquisa foram Scopus, Web of Science, Science Direct, IEEE e Scielo. Essa estratégia de pesquisa e as combinações entre as palavras-chave estão apresentadas no Quadro 1.

Quadro 1- Estratégia para pesquisa nos bancos de dados

\begin{tabular}{|c|c|c|c|c|}
\hline & \multicolumn{2}{|c|}{ Eixos Temáticos } & \multirow{2}{*}{ Combinações } & \multirow{2}{*}{$\begin{array}{c}\text { Bases de } \\
\text { Dados }\end{array}$} \\
\hline & Casa Inteligente & Usuário & & \\
\hline گે & $\begin{array}{l}\text { Smart home } \\
\text { Smart house } \\
\text { Intelligent home } \\
\text { Intelligent house }\end{array}$ & $\begin{array}{l}\text { adopt }^{*} \\
\text { accept }^{*}\end{array}$ & $\begin{array}{c}\text { ("smart home" OR "smart house" OR } \\
\text { "intelligent home" OR "intelligent house") } \\
\text { AND } \\
\text { ("adopt*" OR "accept*") }\end{array}$ & $\begin{array}{l}\text { Scopus } \\
\text { Web of Science } \\
\text { Science Direct } \\
\text { IEEE } \\
\text { Scielo }\end{array}$ \\
\hline
\end{tabular}

Após as definições do Quadro1, foram definidos os seguintes critérios de inclusão e exclusão de artigos no portfólio de estudos: (i) o estudo deve abordar o tema de casa inteligente; (ii) o estudo deve apresentar características ou fatores pertinentes à adoção e/ou aceitação da casa inteligente; (iii) o estudo deve ser um artigo científico completo publicado em revista científica revisada por pares; (iv) o estudo não pode ser artigo ou resumo publicado em conferências, congressos ou semelhantes; (v) o estudo deve ter sido publicado no idioma inglês em periódicos indexados nas bases de dados Scopus, Web of Science, Science Direct, IEEE. Este último critério de seleção, que trata do idioma em que o estudo foi publicado, especifica-se as bases de estudos para o idioma inglês. Na base de dados Scielo realizou-se uma busca nas mesmas condições, porém no idioma português. Essa questão, deve-se ao fato da importância em identificar estudos na literatura sobre o tema de pesquisa no Brasil, país onde está sendo desenvolvido este trabalho. 
Definido o protocolo de revisão, realizou-se uma busca em agosto de 2020 e obteve-se um retorno de 820 artigos. Foi analisada uma amostra com 10 artigos, e os estudos apresentaram uma boa aderência à pesquisa e, portanto, validadas as palavras-chaves de busca.

A fase de condução da pesquisa relata os procedimentos usados para identificação da pesquisa, seleção de estudos primários, extração e monitoramento de dados e a síntese de dados, Figura 1.

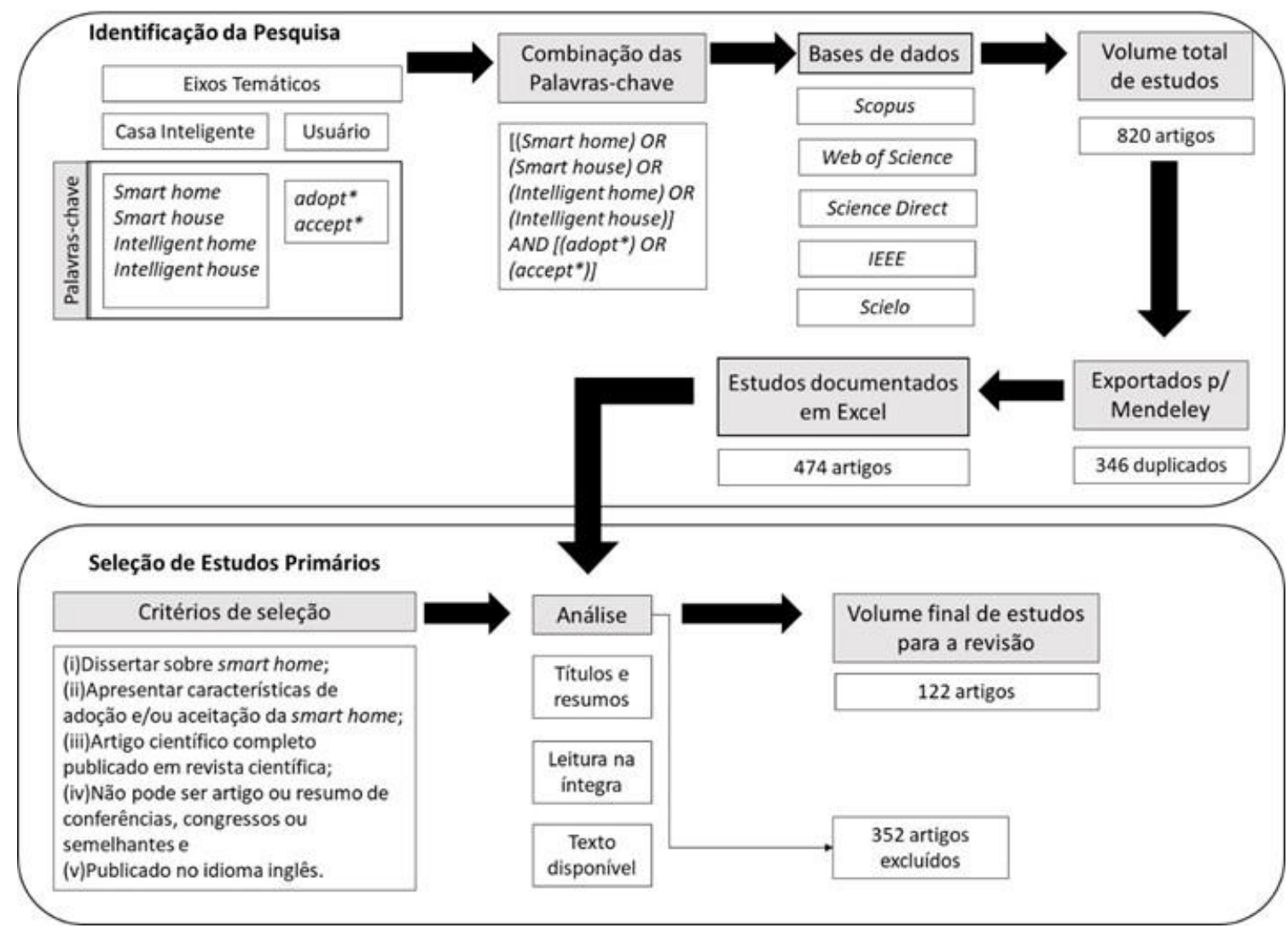

Figura 1 - Fase de condução da pesquisa do processo de revisão

A pesquisa com as palavras-chave e termos, foi realizada utilizando os campos título, resumo e palavras-chave dos artigos publicados nas bases de dados, considerando apenas publicações em periódicos científicos realizadas até 2020. Foram encontrados 820 artigos que foram exportados para o software Mendeley ${ }^{\circledR}$. Foram excluídas 346 publicações duplicadas diretamente pelo software e publicações de conferências, livros e capítulos de livros, obtendo, 474 artigos. As informações dos 474 artigos foram exportadas para planilha eletrônica (Excel®) para o controle da condução das próximas etapas. Os critérios de seleção definidos no protocolo de revisão foram utilizados para análise dos títulos, resumos e dos textos na íntegra. Após essa análise 351 artigos foram excluídos por não atenderem aos critérios e, portanto, 122 artigos foram incluídos no portfólio final de estudos primários para o 
desenvolvimento da revisão. Foram considerados fora de escopo todos os artigos que não atendiam os critérios determinados no protocolo no processo de planejamento desta revisão, ou seja, todos os artigos que não apresentavam adoção ou aceitação de casa inteligente em seu

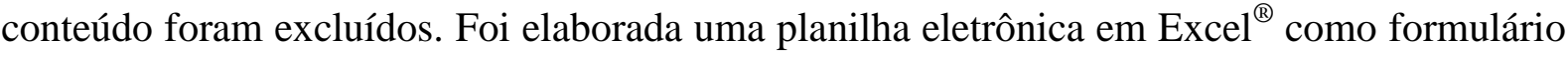
para armazenar as informações obtidas por meio da leitura dos artigos. Assim, é possível sintetizar os dados, próxima atividade da revisão sistemática, e fornecer as informações necessárias para a análise bibliométrica e análise de conteúdo.

$\mathrm{Na}$ fase de relato da pesquisa, os resultados da revisão de literatura são apresentados em duas seções, a seguir. A primeira apresenta uma síntese quantitativa da literatura sobre o tema, sendo denominada de análise bibliométrica, onde foram consideradas diversas variáveis, tais como instituições de pesquisa, periódicos e países de estudos. A segunda seção apresenta um relato e análise qualitativa do conteúdo apresentado nos 122 artigos integrados nesta revisão, baseadas nas características desses artigos.

\section{ANÁLISE QUANTITATIVA}

O portfólio de estudos é composto por 122 artigos, dos quais 31 são estudos teóricos apresentados por meio de uma revisão sistemática ou modelo conceitual e os demais, 91 artigos, são estudos empíricos. Um estudo desenvolvido por Filippini (1997) apresenta uma compilação sobre os métodos de pesquisa utilizados na área de gestão de operações. A Figura 2 apresenta, por meio da tipologia de métodos de pesquisa proposta por Filippini (1997), como os estudos empíricos estão distribuídos.
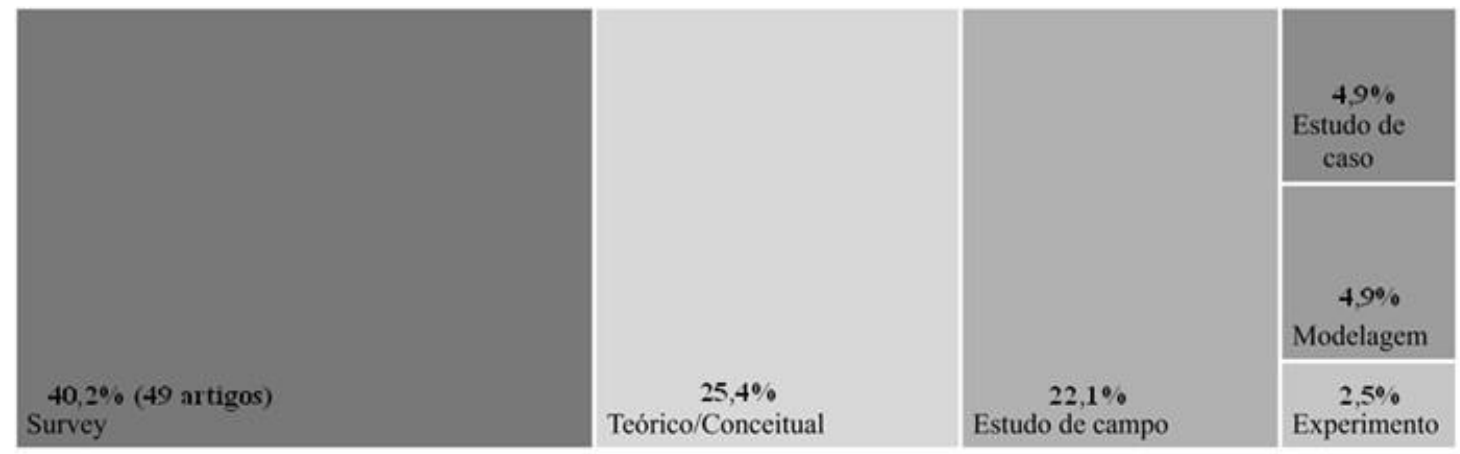

Figura 2 - Distribuição dos estudos do portfólio de pesquisa

O portfólio de estudos pode ser analisado sob a frequência de distribuição dos periódicos nos quais os artigos foram publicados (Figura 3). Os 122 artigos estão distribuídos em 91 periódicos identificados nesta pesquisa. 


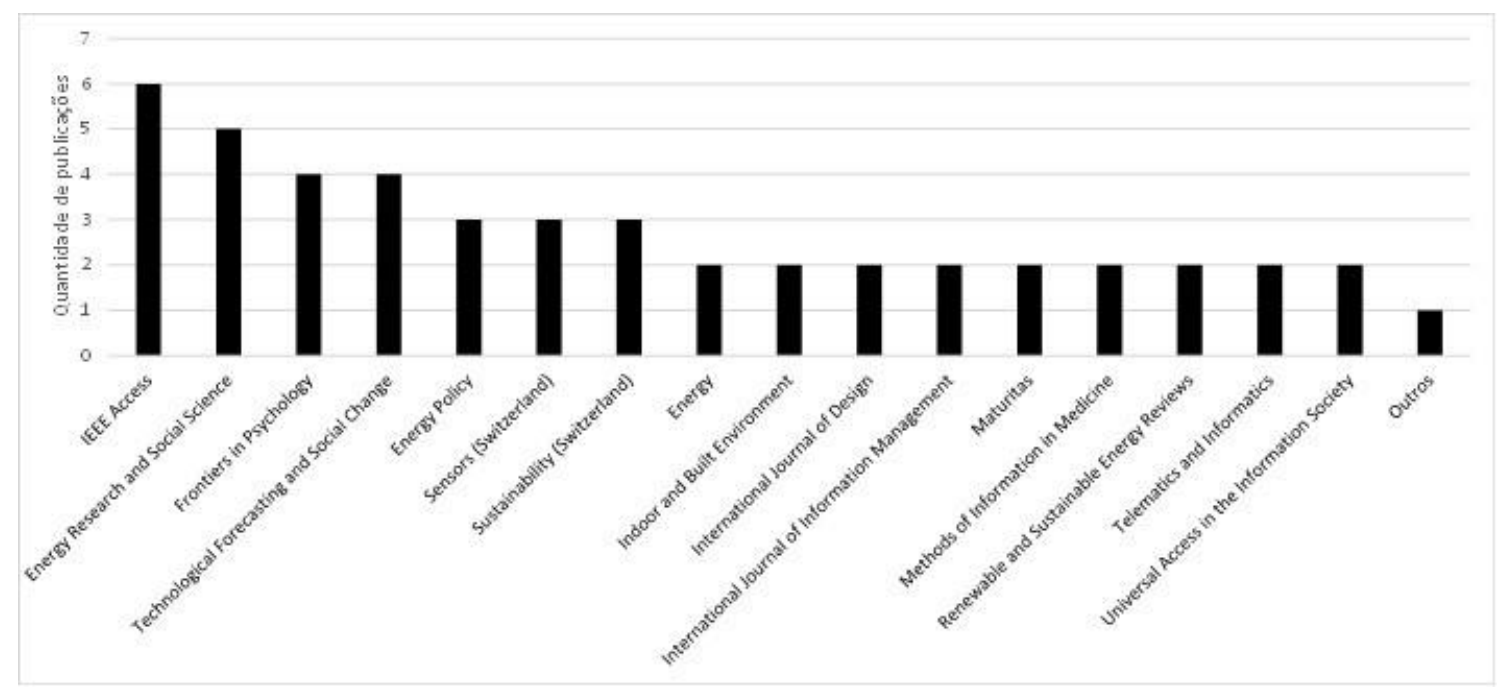

Figura 3 - Periódicos com publicações selecionadas para a revisão

Observa-se que o tema de pesquisa é multidisciplinar, permeando periódicos científicos de diversas áreas como Engenharia, Energia, Ciências Sociais, Psicologia, Negócios, Gestão e Contabilidade, Ciência Ambiental, Física, Engenharia Elétrica, Matemática, Medicina, Profissões da saúde, Ciência da Computação (Tabela 1). Dos 91 diferentes periódicos com artigos publicados no portfólio, 75 destes periódicos apresentam, na análise bibliométrica apenas 1 artigo. Os periódicos com maiores frequências representam menos de $20 \%$ do total identificado. As áreas de Energia e Ciências Sociais possuem maior quantidade de periódicos, três cada. A área de Engenharia se destaca pela maior frequência absoluta no periódico IEEE Access. Neste último periódico foi possível encontrar estudos que apresentam revisão sistemática da literatura sobre monitoramento de tecnologias em casa inteligente baseado em internet das coisas (Internet of Things - IoT) (e.g., MASWADI; GHANI; HAMID, 2020).

Tabela 1 - Características dos periódicos identificados na pesquisa e classificação segundo a base Scopus 


\begin{tabular}{|c|c|c|c|}
\hline Periódico & $\begin{array}{c}\text { Frequência } \\
\text { absoluta }\end{array}$ & Área(s) & SJR \\
\hline IEEE Access & 6 & Engenharia & 0,775 \\
\hline Energy Research and Social Science & 5 & Energia e Ciências Sociais & 2,205 \\
\hline Frontiers in Psychology & 4 & Psicologia & 0,914 \\
\hline Technological Forecasting and Social Change & 4 & $\begin{array}{l}\text { Negócios, Gestão e } \\
\text { Contabilidade }\end{array}$ & 1,815 \\
\hline Energy Policy & 3 & $\begin{array}{l}\text { Energia e Ciência } \\
\text { Ambiental }\end{array}$ & 2,168 \\
\hline Sensors & 3 & $\begin{array}{l}\text { Física e Engenharia } \\
\text { Elétrica }\end{array}$ & 0,653 \\
\hline Sustainability & 3 & Ciências Sociais & 0,581 \\
\hline Energy & 2 & $\begin{array}{c}\text { Matemática e Ciência } \\
\text { ambiental }\end{array}$ & 2,166 \\
\hline Indoor and Built Environment & 2 & Medicina & 0,43 \\
\hline International Journal of Design & 2 & $\begin{array}{l}\text { Negócios, Gestão e } \\
\text { Contabilidade }\end{array}$ & 0,72 \\
\hline $\begin{array}{l}\text { International Journal of Information } \\
\text { Management }\end{array}$ & 2 & Ciências Sociais & 2,881 \\
\hline Maturitas & 2 & Medicina & 1,189 \\
\hline Methods of Information in Medicine & 2 & Profissões da saúde & 0,588 \\
\hline Renewable and Sustainable Energy Reviews & 2 & Energia & 3,632 \\
\hline Telematics and Informatics & 2 & Ciência da Computação & 1,441 \\
\hline Universal Access in the Information Society & 2 & Ciência da Computação & 0,486 \\
\hline
\end{tabular}

Outro resultado da análise bibliométrica está relacionado à localização dos pesquisadores e aos locais onde os estudos empíricos foram realizados sobre o tema de pesquisa. As instituições de pesquisa dos autores do portfólio estão distribuídas em 34 países, em cinco continentes (Tabela 2). Alguns artigos são realizados em parceria com mais de um autor de instituições diferentes, sendo estas instituições localizadas, muitas vezes, em países diferentes. Foram contabilizadas as instituições sede de cada um dos autores do artigo. A localização das instituições de origem de todos os autores dos artigos, também, foi contabilizada neste levantamento. 
Tabela 2 - Países e continentes das instituições dos autores

\begin{tabular}{|c|c|c|c|}
\hline Continente & País & $\mathrm{N}^{0}$ de artigos & Frequência \\
\hline Europa & Reino Unido & 19 & $11,7 \%$ \\
\hline \multirow[t]{14}{*}{$(40,1 \%)$} & Alemanha & 9 & $5,6 \%$ \\
\hline & Itália & 6 & $3,7 \%$ \\
\hline & França & 6 & $3,7 \%$ \\
\hline & Finlândia & 4 & $2,5 \%$ \\
\hline & Suécia & 4 & $2,5 \%$ \\
\hline & Holanda & 4 & $2,5 \%$ \\
\hline & Espanha & 2 & $1,2 \%$ \\
\hline & República Tcheca & 2 & $1,2 \%$ \\
\hline & Dinamarca & 2 & $1,2 \%$ \\
\hline & Áustria & 2 & $1,2 \%$ \\
\hline & Polônia & 2 & $1,2 \%$ \\
\hline & Eslovênia & 1 & $0,6 \%$ \\
\hline & Bulgária & 1 & $0,6 \%$ \\
\hline & Grécia & 1 & $0,6 \%$ \\
\hline Ásia & Coreia & 16 & $9,9 \%$ \\
\hline \multirow[t]{13}{*}{$(33,3 \%)$} & China & 8 & $4,9 \%$ \\
\hline & Malásia & 4 & $2,5 \%$ \\
\hline & Taiwan & 4 & $2,5 \%$ \\
\hline & Tailândia & 4 & $2,5 \%$ \\
\hline & Jordânia & 3 & $1,9 \%$ \\
\hline & Japão & 2 & $1,2 \%$ \\
\hline & Irã & 2 & $1,2 \%$ \\
\hline & Turquia & 2 & $1,2 \%$ \\
\hline & Emirados Árabes & 2 & $1,2 \%$ \\
\hline & Singapura & 2 & $1,2 \%$ \\
\hline & Israel & 2 & $1,2 \%$ \\
\hline & Arábia Saudita & 2 & $1,2 \%$ \\
\hline & Índia & 1 & $0,6 \%$ \\
\hline América & USA & 27 & $16,7 \%$ \\
\hline \multirow[t]{2}{*}{$(20,4 \%)$} & Canadá & 4 & $2,5 \%$ \\
\hline & Brasil & 2 & $1,2 \%$ \\
\hline $\begin{array}{c}\text { Oceania } \\
(6 \%)\end{array}$ & Austrália & 9 & $5,6 \%$ \\
\hline $\begin{array}{c}\text { África } \\
(1 \%)\end{array}$ & Marrocos & 1 & $0,6 \%$ \\
\hline
\end{tabular}

O continente com maior expressividade é a Europa que corresponde a 40,1\% das instituições sede dos autores dos artigos deste portfólio. Entre os diversos países, o Reino Unido é o país sede que possui a maior quantidade de artigos, com representatividade de $11,7 \%$ do total. $\mathrm{O}$ continente asiático também possui uma representatividade como sede dos pesquisadores sobre o tema, atingindo 33,3\% das instituições de pesquisa sede dos pesquisadores. Apesar da América ser um continente com menor número de pesquisas sobre o tema em relação a Europa e Ásia, possui o país com maior representatividade, os Estados Unidos, com 16,7\% das pesquisas sobre o tema no portfólio. 


\section{ANÁLISE DE CONTEÚDO}

\subsection{Benefícios percebidos pelos usuários sobre a casa inteligente}

A literatura tem apresentado fatores que conduzem a aceitação da casa inteligente pelos usuários. Uma parte desses fatores tem sido discutida na literatura como benefícios potenciais e percebidos que a casa inteligente pode oferecer como vantagens para os usuários (FRIEDEWALD et al., 2005). Na literatura recente, foi observado que os benefícios que a casa inteligente proporciona também podem ser identificados por meio de quatro diferentes grupos: (i) benefícios ambientais, (ii) financeiros, (iii) psicológicos e (iv) relacionados à saúde (MARIKYAN; PAPAGIANNIDIS; ALAMANOS, 2019).

Os benefícios ambientais que a casa inteligente promove podem ser classificados como de curto ou longo prazo ao usuário. Entre os benefícios ambientais de curto prazo são mencionados a redução do uso de energia, obtenção de feedback sobre o consumo de energia e sugestões de como usar a energia de forma eficiente em uma casa (e.g., BHATI; HANSEN; CHAN, 2017; NILSSON et al., 2018). Entre os benefícios ambientais a longo prazo como a sustentabilidade ambiental e redução das emissões de carbono (e.g., SCHILL; GODEFROITWINKEL; BARBAROSSA, 2019).

Os benefícios financeiros possibilitam obter menores custos com as atividades de rotina em uma casa, ou obter acesso a saúde, devido a custos menores com consultas médicas virtuais, ou ainda, promover um consumo sustentável (e.g., RAJAGOPAL et al., 2019; SCHIEWECK et al., 2018).

Entre os benefícios psicológicos são mencionados a possibilidade de promover o entretenimento, permitir a interação virtual, causar o bem-estar e melhorar o conforto das pessoas em casa (e.g., YANG; LEE; ZO, 2017).

Em relação aos benefícios associados a saúde, pode ser mencionada a maior acessibilidade e disponibilidade de cuidados em casa, a segurança que o usuário pode ter em casa, a conexão social e comunicação com o ambiente externo a casa, a possibilidade de detecção de eventos de risco de vida, a redução de erros médicos e o bem-estar do envelhecimento e das pessoas vulneráveis (e.g., COURTNEY, 2008; RIALLE et al., 2008).

Diante dessa abordagem sobre os benefícios que a casa inteligente pode oferecer ao usuário, por meio dos quatro grupos de benefícios propostos na literatura (MARIKYAN; PAPAGIANNIDIS; ALAMANOS, 2019), este trabalho reporta a frequência que os benefícios 
são apresentados nos estudos que compõem o portfólio desta revisão (Tabela 3). Nessa análise, observou-se que um mesmo artigo pode apresentar mais de um tipo de benefício.

Tabela 3 - Benefícios potenciais e percebidos pelo usuário na adoção de uma casa inteligente

\begin{tabular}{|c|c|c|c|}
\hline Benefícios & $\begin{array}{l}\text { Frequência } \\
\text { na literatura }\end{array}$ & Vantagens & $\begin{array}{l}\text { Frequência } \\
\text { na literatura }\end{array}$ \\
\hline \multirow{6}{*}{ Saúde } & \multirow{6}{*}{62} & Acessibilidade e disponibilidade de cuidados & 23 \\
\hline & & Segurança do usuário & 33 \\
\hline & & Conectividade social e comunicação & 17 \\
\hline & & Detecção de eventos de risco de vida & 7 \\
\hline & & Redução de erros médicos & 12 \\
\hline & & $\begin{array}{l}\text { Bem-estar do envelhecimento e pessoas } \\
\text { vulneráveis }\end{array}$ & 22 \\
\hline \multirow{5}{*}{ Ambiental } & \multirow{5}{*}{45} & Redução do uso de energia & 31 \\
\hline & & Feedback sobre o consumo & 18 \\
\hline & & $\begin{array}{l}\text { Sugestões de como usar a energia de forma } \\
\text { eficiente }\end{array}$ & 15 \\
\hline & & Sustentabilidade ambiental & 14 \\
\hline & & Redução das emissões de carbono & 3 \\
\hline \multirow{3}{*}{ Financeiro } & \multirow{3}{*}{21} & Menores custos em consultas virtuais & 11 \\
\hline & & Acessibilidade aos cuidados de saúde & 12 \\
\hline & & Consumo sustentável & 1 \\
\hline \multirow{7}{*}{ Psicológico } & \multirow{7}{*}{55} & Entretenimento & 21 \\
\hline & & Interação virtual & 6 \\
\hline & & Superação à sensação de isolamento & 4 \\
\hline & & Bem-estar e conforto & 30 \\
\hline & & Inclusão social & 12 \\
\hline & & Economia de trabalho & 6 \\
\hline & & Home office & 1 \\
\hline
\end{tabular}

\subsection{Barreiras para adoção da casa inteligente}

A adoção pelos usuários e a difusão no mercado da casa inteligente tem se apresentado de forma lenta, apesar dos potenciais benefícios apresentados (BALTA-OZKAN et al., 2013a). A maior aceitação da casa inteligente depende de alguns fatores (HANNOVER; OSTWESTFALEN-LIPPE; HANNOVER, 2020), esses fatores são as possíveis barreiras que podem dificultar a implementação das casas inteligentes (MARIKYAN; PAPAGIANNIDIS; ALAMANOS, 2019).

A literatura recente sobre a casa inteligente pela perspectiva do usuário indica que principais barreiras para a adoção da casa inteligente podem ser categorizadas como: (i) tecnológicas, (ii) financeira, ética e legal e relacionadas à (iii) falta de conhecimento e resistência 
psicológica (MARIKYAN; PAPAGIANNIDIS; ALAMANOS, 2019). As barreiras identificadas nos artigos que compõem o portfólio de estudos estão apresentadas na Tabela 4. Este trabalho adotou a categorização de barreiras para a adoção de casa inteligente, proposta e apresentada no estudo de Marikyan; Papagiannidis; Alamanos, (2019) para análise dos estudos do portfólio. No grupo de barreiras tecnológicas estão incluídos fatores como segurança, usabilidade, invasão de privacidade, confiabilidade, complexidade e interoperabilidade. A adequação da tecnologia tem sido considerado um dos fatores mais importantes no desenvolvimento da casa inteligente (BALTA-OZKAN et al., 2013b). Alguns estudos de adoção de casas inteligentes têm aumentado o foco nos recursos da tecnologia que podem representar ameaças aos usuários e influenciar a percepção da tecnologia o processo de adoção (HEINZ et al., 2013; KIM et al., 2019).

Tabela 4 - Barreiras para adoção de uma casa inteligente

\begin{tabular}{clll}
\hline Barreiras & $\begin{array}{l}\text { Frequência } \\
\text { na literatura }\end{array}$ & Fatores & $\begin{array}{l}\text { Frequência } \\
\text { na literatura }\end{array}$ \\
\hline & & Segurança & 36 \\
& & Usabilidade & 22 \\
Tecnológicas & 72 & Invasão de privacidade & 48 \\
& & Confiabilidade & 22 \\
& & Complexidade (Interoperabilidade) & 24 \\
& & Transição tecnológica & 6 \\
\hline & Preço & 16 \\
Financeira, & Custo de instalação & 16 \\
ética e legal & 43 & Custo de reparo e manutenção & 9 \\
& & Preocupação com o uso indevido de dados privados & 24 \\
& & Consentimento de idosos e pacientes & 6 \\
& & Falta de conduta legal & 6 \\
Gap do & & Conflitos de regulamentação & 8 \\
conhecimento & \multirow{2}{*}{$\begin{array}{c}\text { esistência } \\
\text { psicológica }\end{array}$} & Barreira humana & 13 \\
\hline & Resistência ao uso de tecnologia inovadora & 5 \\
& & Falta de conhecimento prévio ou experiência & 8 \\
\hline & Crenças sociais & 5 \\
\hline
\end{tabular}

Outro grupo de barreiras analisado neste trabalho envolve fatores financeiros, éticos e legislativos. As barreiras financeiras estão relacionadas com o preço da tecnologia, e com os custos de instalação, reparo e manutenção, que podem desmotivar o usuário na adoção da casa inteligente (BALTA-OZKAN; BOTELER; AMERIGHI, 2014; MAMONOV; KOUFARIS, 2020). As barreiras éticas estão relacionadas com o fator de segurança e privacidade dos dados, devido a capacidade da casa inteligente em coletar e armazenar dados privados de seus 
usuários (JACOBSSON; BOLDT; CARLSSON, 2016; YANG; LEE; ZO, 2017). As barreiras relacionadas à legislação remetem a uma falta de conduta, ou leis, que regulamentam o uso da tecnologia nas casas inteligentes (WONG; LEUNG, 2016).

O último grupo de barreias retrata a falta de conhecimento prévio e a resistência psicológica apresentada pelo usuário da casa inteligente. Como as tecnologias da casa inteligente são tecnologias emergentes, os usuários não estão totalmente cientes de suas funções, riscos e benefícios potenciais (MARIKYAN; PAPAGIANNIDIS; ALAMANOS, 2019). A baixa percepção de utilidade resulta para o usuário uma sensação de perda de controle sobre a tecnologia, ou seja, uma perda de controle da própria casa, o que pode gerar uma resistência em aceitar a casa inteligente (SCHIEWECK et al., 2018). A falta de conhecimento prévio sobre as tecnologias da casa inteligente pode dificultar a difusão de casas inteligentes no mercado, como mostram os estudos de (e.g., MEEKS; SWEANEY; SWAGLER, 1992; TONKIN et al., 2018).

\section{CONSIDERAÇÕES FINAIS}

Este artigo utilizou a revisão sistemática da literatura, baseado no método proposto por Kitchenham (2004), para identificar os benefícios promovidos pela casa inteligente e as barreiras do processo de adoção da casa inteligente.

Os benefícios relacionados à saúde tem sido um dos mais mencionados na literatura. Esses benefícios apresentam a possibilidade do monitoramento dos usuários da casa inteligente, como os idosos, por meio de sensores de movimento, câmeras, entre outras tecnologias. Esse monitoramento permite a realização de uma comunicação rápida com parentes ou médicos quando necessário ou, ainda, gerar um histórico de dados que permite um diagnóstico mais assertivo sobre possíveis enfermidades. Os benefícios ambientais têm sido um dos mais motivadores entre os usuários que possuem uma preocupação com o meio ambiente, sustentabilidade. Pois, por meio da casa inteligente, é possível o gerenciamento de energia da casa, controlando e monitorando o uso de aparelhos domésticos de forma a evitar desperdícios e obter uma forma mais eficiente do uso da energia em casa.

Neste trabalho, fica evidente que as barreiras tecnológicas são preponderantes no processo de adoção das casas inteligentes. A complexidade da tecnologia pode ser um fator determinante para a percepção da usabilidade pelo usuário. Outra questão associada a tecnologia está relacionada à segurança, ou seja, à preocupação do usuário em relação a invasão de privacidade e roubo de dados. 
Esse artigo contribui para a comunidade acadêmica com uma discussão atualizada sobre adoção da casa inteligente. Para a comunidade prática, este artigo facilita criação de estratégias para disseminação da casa inteligente no mercado.

\section{REFERÊNCIAS}

BALTA-OZKAN, N.; BOTELER, B.; AMERIGHI, O. European smart home market development: Public views on technical and economic aspects across the United Kingdom, Germany and Italy. Energy Research and Social Science, vol. 3, no. C, p. 65-77, 2014. https://doi.org/10.1016/j.erss.2014.07.007.

BALTA-OZKAN, N.; DAVIDSON, R.; BICKET, M.; WHITMARSH, L. Social barriers to the adoption of smart homes. Energy Policy, vol. 63, p. 363-374, 2013a. https://doi.org/10.1016/j.enpol.2013.08.043.

BALTA-OZKAN, N.; DAVIDSON, R.; BICKET, M.; WHITMARSH, L. The development of smart homes market in the UK. Energy, vol. 60, p. 361-372, 2013b. https://doi.org/10.1016/j.energy.2013.08.004.

BHATI, Abhishek; HANSEN, Michael; CHAN, Ching Man. Energy conservation through smart homes in a smart city: A lesson for Singapore households. Energy Policy, vol. 104, p. 230-239, 2017. DOI https://doi.org/10.1016/j.enpol.2017.01.032.

Available

at: http://www.sciencedirect.com/science/article/pii/S0301421517300393.

CHAN, Marie; CAMPO, Eric; ESTÈVE, Daniel; FOURNIOLS, Jean-Yves. Smart homes - Current features and future perspectives. Maturitas, vol. 64, p. 90-97, 2009. Available at: https://pdf.sciencedirectassets.com/271192/1-s2.0-S0378512209X00125/1-s2.0-

S0378512209002606/main.pdf?X-Amz-Security-

Token=AgoJb3JpZ2luX2VjEJr\%2F\%2F\%2F\%2F\%2F\%2F\%2F\%2F\%2F\%2FwEaCXVzLWVhc3QtMSJGMEQ CIDTk4a8aG3ab3n0byb4IPV62hbHZDk\%2FOK7RYkiIgZhWvAiA3vh4tAvweuU. Accessed on: 16 Sep. 2019.

COURTNEY, K.L. Privacy and senior willingness to adopt smart home information technology in residential care facilities. Methods of Information in Medicine, vol. 47, no. 1, p. 76-81, 2008. https://doi.org/10.3414/ME9104.

DONG, Xuebing; CHANG, Yaping; WANG, Yawei; YAN, Jun. Understanding usage of Internet of Things (IOT) systems in China Cognitive experience and affect experience as moderator. INFORMATION TECHNOLOGY \& PEOPLE, vol. 30, no. 1, p. 117-138, 2017. https://doi.org/10.1108/ITP-11-2015-0272.

FILIPPINI, Roberto. Operations management research: Some reflections on evolution, models and empirical studies in OM. International Journal of Operations and Production Management, vol. 17, no. 7, p. 655-670, 1997. https://doi.org/10.1108/01443579710175583.

FRIEDEWALD, M.; DA COSTA, O.; PUNIE, Y.; ALAHUHTA, P.; HEINONEN, S. Perspectives of ambient intelligence in the home environment. Telematics and Informatics, vol. 22, no. 3, p. 221-238, 2005. https://doi.org/10.1016/j.tele.2004.11.001.

FURSZYFER DEL RIO, D.D.; SOVACOOL, B.K.; BERGMAN, N.; MAKUCH, K.E. Critically reviewing smart home technology applications and business models in Europe. Energy Policy, vol. 144, no. 111631, 2020.

GRAM-HANSSEN, Kirsten; DARBY, Sarah J. "Home is where the smart is"? Evaluating smart home research and approaches against the concept of home. Energy Research and Social Science, vol. 37, p. 94-101, 1 Mar. 2018. https://doi.org/10.1016/j.erss.2017.09.037.

HANNOVER, Leibniz Universtät; OSTWESTFALEN-LIPPE, Technische Hochschule; HANNOVER, Leibniz Universität. Smart home in private households: Status quo, discussion, and new insights. International Journal of Service Science, Management, Engineering, and Technology, vol. 11, no. 4, p. 122-136, 2020. .

HEINZ, Melinda; MARTIN, Peter; MARGRETT, Jennifer A; YEARNS, Mary; FRANKE, Warren; YANG, Hen-I; WONG, Johnny; CHANG, Carl K. Perceptions of Technology among Older Adults. JOURNAL OF GERONTOLOGICAL NURSING, vol. 39, no. 1, p. 42-51, Jan. 2013. https://doi.org/10.3928/0098913420121204-04.

JACOBSSON, A.; BOLDT, M.; CARLSSON, B. A risk analysis of a smart home automation system. Future Generation Computer Systems, vol. 56, p. 719-733, 2016. https://doi.org/10.1016/j.future.2015.09.003. 
KANG, Ziqiu; CATAL, Cagatay; TEKINERDOGAN, Bedir. Machine learning applications in production lines: A systematic literature review. Computers and Industrial Engineering, vol. 149, p. 106773, 1 Nov. 2020. https://doi.org/10.1016/j.cie.2020.106773.

KIM, Dongyeon; PARK, Kyuhong; PARK, Yongjin; AHN, Jae-Hyeon. Willingness to provide personal information: Perspective of privacy calculus in IoT services. COMPUTERS IN HUMAN BEHAVIOR, vol. 92, p. 273-281, Mar. 2019. https://doi.org/10.1016/j.chb.2018.11.022.

KITCHENHAM, Barbara. Procedures for Performing Systematic Reviews Kitchenham, B., 2004. Keele, UK, Keele University, vol. 33, no. 2004, p. 1-26, 2004. .

LIU, Yu. Simulation of art design of indoor furnishings based on FPGA and internet of things system. Microprocessors and Microsystems, vol. 83, p. 104023, 1 Jun. 2021. DOI 10.1016/j.micpro.2021.104023. Available at: https://linkinghub.elsevier.com/retrieve/pii/S0141933121001964. Accessed on: 20 Jan. 2021.

MAMONOV, S.; KOUFARIS, M. Fulfillment of higher-order psychological needs through technology: The case of smart thermostats. International Journal of Information Management, vol. 52, 2020. https://doi.org/10.1016/j.ijinfomgt.2020.102091.

MARIKYAN, D.; PAPAGIANNIDIS, S.; ALAMANOS, E. A systematic review of the smart home literature: A user perspective. Technological Forecasting and Social Change, vol. 138, p. 139-154, 2019. https://doi.org/10.1016/j.techfore.2018.08.015.

MASWADI, K.; GHANI, N.B.A.; HAMID, S.B. Systematic Literature Review of Smart Home Monitoring Technologies Based on IoT for the Elderly. IEEE Access, vol. 8, p. 92244-92261, 2020. https://doi.org/10.1109/ACCESS.2020.2992727.

MEEKS, C.B.; SWEANEY, A.L.; SWAGLER, R.M. Adopting New Technology: The Case of Electrical Plugs and Receptacles. Home Economics Research Journal, vol. 20, no. 4, p. 264-274, 1992. https://doi.org/10.1177/1077727X9202000403.

MUSAWIR, Ata ul; ABD-KARIM, Saipol Bari; MOHD-DANURI, Mohd Suhaimi. Project governance and its role in enabling organizational strategy implementation: A systematic literature review. International Journal of Project Management, vol. 38, no. 1, p. 1-16, 1 Jan. 2020. https://doi.org/10.1016/j.ijproman.2019.09.007.

NILSSON, Anders; WESTER, Misse; LAZAREVIC, David; BRANDT, Nils. Smart homes, home energy management systems and real-time feedback: Lessons for influencing household energy consumption from a Swedish field study. Energy and Buildings, vol. 179, p. 15-25, 2018 . DOI https://doi.org/10.1016/j.enbuild.2018.08.026. http://www.sciencedirect.com/science/article/pii/S0378778818311691.

RAJAGOPAL, K.; MAHAJAN, V.; SEN, S.; DIVKAR, S. Energy efficient smart home automation adoption-A research. International Journal of Innovative Technology and Exploring Engineering, vol. 8, no. 11 Special, p. 536-540, 2019. https://doi.org/10.35940/ijitee.K1090.09811S19.

RIALLE, V; OLLIVET, C; GUIGUI, C; HERVE, C. What do family caregivers of Alzheimer's disease patients desire in smart home technologies? METHODS OF INFORMATION IN MEDICINE, vol. 47, no. 1, p. 6369, 2008. https://doi.org/10.3414/ME9102.

SCHIEWECK, Alexandra; UHDE, Erik; SALTHAMMER, Tunga; SALTHAMMER, Lea C; MORAWSKA, Lidia; MAZAHERI, Mandana; KUMAR, Prashant. Smart homes and the control of indoor air quality. Renewable and Sustainable Energy Reviews, vol. 94, p. 705-718, 2018. DOI 10.1016/j.rser.2018.05.057. Available at: https://doi.org/10.1016/j.rser.2018.05.057. Accessed on: 27 Aug. 2019.

SCHILL, Marie; GODEFROIT-WINKEL, Delphine; BARBAROSSA, Camilla. Consumers' intentions to purchase smart home objects: Do environmental issues matter? Ecological Economics, vol. 161, p. 176-185, 2019. DOI 10.1016/j.ecolecon.2019.03.028. Available at: https://doi.org/10.1016/j.ecolecon.2019.03.028. Accessed on: 27 Aug. 2019.

SEPASGOZAR, Samad; KARIMI, Reyhaneh; FARAHZADI, Leila; MOEZZI, Farimah; SHIROWZHAN, Sara; EBRAHIMZADEH, Sanee M; HUI, Felix; AYE, Lu. A Systematic Content Review of Artificial Intelligence and the Internet of Things Applications in Smart Home. APPLIED SCIENCES-BASEL, vol. 10, no. 9, 2020. https://doi.org/10.3390/app10093074.

SILVA, Philippe Barbosa; ANDRADE, Michelle; FERREIRA, Sara. Machine learning applied to road safety modeling: A systematic literature review. Journal of Traffic and Transportation Engineering (English 
Edition), vol. 7, no. 6, p. 775-790, 1 Dec. 2020. https://doi.org/10.1016/j.jtte.2020.07.004.

SPOLAÔR, Newton; LEE, Huei Diana; TAKAKI, Weber Shoity Resende; ENSINA, Leandro Augusto; COY, Claudio Saddy Rodrigues; WU, Feng Chung. A systematic review on content-based video retrieval. Engineering Applications of Artificial Intelligence, vol. 90, p. 103557, 1 Apr. 2020. https://doi.org/10.1016/j.engappai.2020.103557.

TONKIN, E.L.; BURROWS, A.; WOZNOWSKI, P.R.; LASKOWSKI, P.; YORDANOVA, K.Y.; TWOMEY, N.; CRADDOCK, I.J. Talk, text, tag? Understanding self-annotation of smart home data from a user's perspective. Sensors (Switzerland), vol. 18, no. 7, 2018. https://doi.org/10.3390/s18072365.

WAN, Xiaoyu; WANG, Tingyi; ZHANG, Wei; CAO, Jie. Perceived value of online customization experience in China: Concept, measurement, and consequences. Journal of High Technology Management Research, vol. 28, no. 1, p. 17-28, 1 Jan. 2017. https://doi.org/10.1016/j.hitech.2017.04.002.

WONG, Johnny Kwok Wai; LEUNG, Jodith K L. Modelling factors influencing the adoption of smart-home technologies. FACILITIES, vol. 34, no. 13-14, p. 906-923, 2016. https://doi.org/10.1108/F-05-2016-0048.

YANG, H.; LEE, H.; ZO, H. User acceptance of smart home services: An extension of the theory of planned behavior. Industrial Management and Data Systems, vol. 117, no. 1, p. 68-89, 2017. https://doi.org/10.1108/IMDS-01-2016-0017. 